\title{
Nondestructive Evaluations of Melanin-related Compounds in the Skin Using Electron Paramagnetic Resonance and Permeability Measurements
}

\author{
Kouichi NaKagawa, ${ }^{* \dagger}$ Satoko MinaKawa, ${ }^{* *}$ and Daisuke SaWAmura** \\ *Division of Regional Innovation, Graduate School of Health Sciences, Hirosaki University, 66-1 Hon-cho, \\ Hirosaki 036-8564, Japan \\ **Department of Dermatology, Graduate School of Medicine, Hirosaki University, 5 Zaifu-cho, \\ Hirosaki 036-8562, Japan
}

\begin{abstract}
Melanin-related compounds in paraffin-embedded tissue specimens of basal cell carcinoma (BCC), seborrheic keratosis (SK), malignant melanoma (MM), and nevus pigmentosus (NP) were nondestructively investigated using permeability measurements (light penetration into pigmented lesion), X-band $(9.4 \mathrm{GHz})$ electron paramagnetic resonance (EPR), and EPR imaging (EPRI). The paramagnetic species in BCC, SK, MM, and NP specimens were analyzed using intensity, linewidth, spectral pattern, and X-band EPRI. The EPR spectra of BCC, SK, and NP showed a single line pattern. The EPR signal intensities of the BCC, SK, and NP samples corresponded to the permeability values that are directly related to pigment color tone, except for MM. The correlation coefficient between EPR and permeability was supported by the high degree of linear relation in the range. We further analyzed MM and speculated that MM contains an additional signal of the pheomelanin radical. In MM and NP samples, two-dimensional (2D) EPRI revealed paramagnetic species distribution and different magnitudes. The paramagnetic (radical) species are directly related to the pigmented lesion site. To conclude, spectroscopic analyses suggest that pheomelanin-related compounds may exist in malignant melanoma.
\end{abstract}

Keywords Skin, pigments, permeability, radical, EPR, imaging

(Received January 5, 2020; Accepted January 29, 2020; Advance Publication Released Online by J-STAGE February 7, 2020)

\section{Introduction}

Melanin is a pigment of the skin. ${ }^{1}$ It prevents UV damage to the skin and is associated with involving the development of other skin diseases, such as malignant melanoma (MM). ${ }^{2,3}$ Melanin is composed of eumelanin and pheomelanin. Eumelanin is darker than pheomelanin, which has a red-brownish color. The molecular structures of these two parts are presented in Fig. 1. Melanin loses hydrogen through a biological process and converts into melanin radicals; these radicals are stable. ${ }^{4-6}$ Thus, the melanin pigments also contain melanin radicals, specifically eumelanin and pheomelanin radicals. However, no spectroscopic method other than electron paramagnetic resonance (EPR) or electron spin resonance can help verify the two radicals in the pigments.

X-band $(9.4 \mathrm{GHz})$ EPR imaging (EPRI) has a higher spatial resolution and greater sensitivity than L-band $(1 \mathrm{GHz})$ EPRI. Thus, noninvasive EPRI can provide detailed information on the location and distribution (concentration) of paramagnetic (radical) species in naturally occurring biomedical samples, such as melanin radicals in MM. ${ }^{5-7}$ Melanin (eumelanin) radicals in biomedical samples have been studied using the EPR technique in various ways, such as using paraffin-embedded

$\dagger$ To whom correspondence should be addressed.

E-mail: nakagawa@hirosaki-u.ac.jp human MM and basal cell carcinoma (BCC) specimens. $^{8}$ Melanin radicals in MM and nevus pigmentosus (NP) specimens have also been compared using EPR. ${ }^{5}$ However, melanin radicals in paraffin-embedded various specimens at ambient temperatures have not been characterized using permeability value. Furthermore, ordinary EPR cannot identify the location of radical sites.

Recently, melanin (mostly eumelanin) has been extensively studied; in several studies, melanin radicals in naturally occurring melanin radicals were detected using EPR.5,7,8 However, research on pheomelanin and its radical is limited. Pheomelanin is photosensitive and is decomposed by light,<smiles>CC1=C(O)C(O)=C(C)[C@H]2C=C(C(=O)O)NC12</smiles><smiles>CCC1C(C)=C(O)C2=C(SCCN2)[C@H]1C</smiles>

A portion of eumelanin A portion of pheomelanin

Fig. 1 Parts of the chemical structure of eumelanin and pheomelanin compounds. 
such as UV. ${ }^{9}$ The pheomelanin radical has been found in a case of human redhair and in paraffin-embedded melanoma specimens..$^{5,6}$ Cysteinyldopa (a precursor of pheomelanin) was found in large amounts in the plasma and urine of patients with malignant melanoma. ${ }^{10}$ In addition, the involvement of pheomelanin and its radical in melanoma is currently under discussion. ${ }^{5,10}$

We tried to rule out any relation of melanin-related compounds in the lesion site. We differentiated between eumelanin and pheomelanin radicals in paraffin-embedded human specimens (SK, BCC, MM, and NP) using permeability measurements, EPR, and EPRI. The samples used in this study were specimens that were originally collected for histological examination. Using two-dimensional EPRI, the distribution and relative concentration of the radicals were determined in the samples. In addition, the permeability values of the samples in relation to the EPR intensity were analyzed. We performed an isotropic simulation of the obtained spectrum. Furthermore, we discussed the EPR data in relation to permeability as well as the involvement of skin malignancy-related pheomelanin radicals in melanoma.

\section{Experimental}

\section{Sample preparations}

All protocols used in this study were approved by the Hirosaki University Internal Review Board. ${ }^{11}$ Paraffin-embedded samples were used for histological examination. Thereafter, the paraffinembedded samples were used for the EPR assay and permeability testing. All samples were obtained from patients who had recently visited the dermatology clinic of the Hirosaki University Hospital.

Various intensely colored sections of samples obtained from four SK cases (60-year-old male, 74-year-old male, 104-yearold female, and 93-year-old female) were examined. The paraffinembedded SK samples were used for histological examination. After examination, the samples were used for the EPR assay.

The most intensely colored sections of samples obtained from six nodular BCC cases (87-year-old male, 48-year-old female, 88-year-old female, 76-year-old female, 88-year-old female, and 77-year-old male) were examined. Then, the BCC samples were embedded in paraffin wax for histopathological examination, following which they were used for the EPR assay. Meanwhile, the paraffin-embedded, nonpigmented tissue was used as the control (data not shown).

We also examined the most intensely colored sections of samples obtained from six MM cases (67-year-old male, stage IA; 74-year-old male, stage IIC; 74-year-old male, stage IV; 62-year-old female, stage IB; 65-year-old male, stage IIC; and 69-year-old female, stage IIC) and six NP cases (11-year-old male, 28-year-old female, 34-year-old male; 43-year-old male, 25-year-old male, and 21-year-old male). The MM samples were embedded in paraffin wax for histological examination and then used for the EPR assay. For the control, paraffin-embedded NP sections were used.

\section{Permeability}

The permeability of the samples was measured using a Densitometer Model 301 RS (Fuji Film Co., Ltd., Tokyo, Japan). The light source used was a green light, approximately $570(\mathrm{~nm})$. The same samples were used for the permeability measurements and EPR assay. Sample sizes were approximately $0.2 \mathrm{~mm}$ (thickness). It is notable that if there was no interference of green light, the value was zero. A higher permeability value indicates less permeation in the sample.

\section{EPR settings}

Paraffin-embedded MM and NP samples were examined using the commercially available JEOL RE-3X $9 \mathrm{GHz}$ EPR spectrometer (JEOL Ltd., Tokyo, Japan). Intensely pigmented samples, approximately $3 \times 4 \times 3 \mathrm{~mm}$ each, were cut out from the paraffin-embedded samples to fit into a $5 \mathrm{~mm}$-diameter EPR rod. The static magnetic field was controlled by an electric current without an NMR probe. The paraffin-embedded samples were used for the examination; however, the sample amount varied slightly across patients. The sample was pasted onto an EPR rod and examined using an EPR spectrometer. ${ }^{12}$ All measurements were performed at $21^{\circ} \mathrm{C}$.

\section{EPR imaging settings}

A commercially available JEOL RE-3X $9 \mathrm{GHz}$ EPR spectrometer was modified for use as an EPR imager, to include the attachment of magnetic-field gradient coils (Yonezawa Densen Ltd., Yonezawa, Japan) and their power supplies. For 9.4 GHz EPR imaging, X- and Y-axes of gradient coils with anti-Helmholtz coil configurations were used. Takasago BWS 60-5 bipolar power supplies (Takasago Ltd., Tokyo, Japan) were used. ${ }^{8,13}$

We used 16 equal-angle-spaced projections obtained with a maximum gradient of $\sim 3.3 \mathrm{mT} / \mathrm{cm}$. Before reconstruction, each projection was deconvolved using the measured zero-gradient spectrum to improve the image resolution. The $2 \mathrm{D}$ images were reconstructed using the back-projection algorithm from the EPR-IT software package from the Center for EPR Imaging In Vivo Physiology which is described in earlier literatures. ${ }^{5,14}$

The sample was pasted onto an EPR rod (outer diameter $\sim 4.0 \mathrm{~mm}$ ) for imaging measurements. The rod was positioned in the center of the microwave cavity. For each measurement, the microwave power was selected with the linear section of the power intensity curve. Amplitude modulation values were chosen in such a way that they did not induce any signal distortion, and were always limited to the linewidth value.

\section{Results and Discussion}

First, ordinary EPR measurements were obtained. Figure 2 shows the EPR spectra of paraffin-embedded SK and BCC samples. The EPR results of g-value and peak-to-peak linewidth are consistent with the previously reported values. ${ }^{5,8,15}$ The EPR results revealed that the peak-to-peak linewidths $\left(\Delta H_{\mathrm{pp}}\right)$ of the paraffin-embedded samples were approximately $0.5-0.6 \mathrm{mT}$; furthermore, the g-value was $2.004-2.005$. Thus, we can compare the EPR spectra observed for all samples using the peak-to-peak intensity. It is note that approximate concentrations of the radicals in the specimens were previously reported. ${ }^{5}$ The calculation of the radical concentration can vary with the cutting of the paraffin-embedded sample region, and in addition, the sample region is normally not uniform (specimen to specimen).

We also performed permeability measurements for the samples. The EPR intensities versus permeability values for SK (๑) and BCC $(\bigcirc)$ samples are plotted in Fig. 3. Here we chose the extended error bars due to the cutting out of a portion of the paraffin-embedded specimens. The plot shows the excellent correlation between the EPR intensity and permeability, which is directly related to the sample color tone.

Figure 4 presents the EPR spectra of (A) MM, (B) MM-1 (another melanoma sample), (C) NP, (D) subtracted spectrum of (C) from (A), and (E) subtracted spectrum of (D) from (A). In order to obtain a good subtraction, we multiplied spectrum (C) by 1.6. The MM EPR spectra show a small shoulder at the 


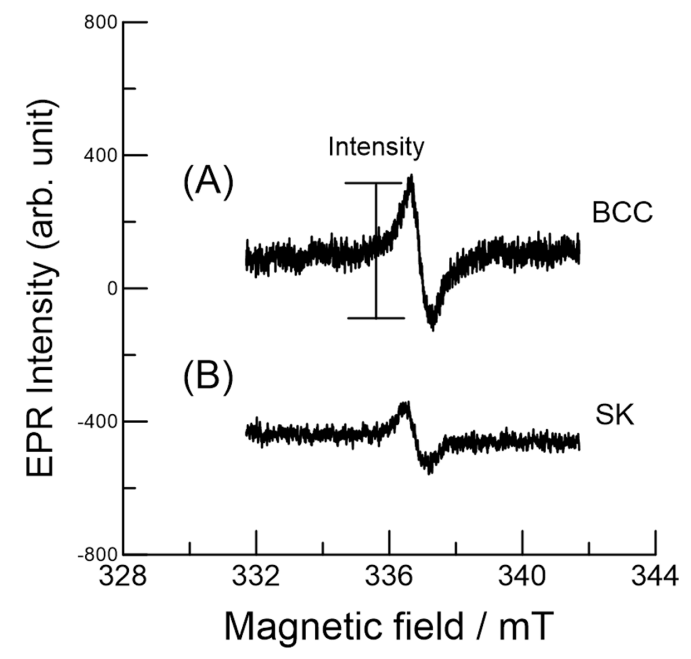

Fig. 2 Representative electron paramagnetic resonance (EPR) spectra of seborrheic keratosis (SK) and basal cell carcinoma (BCC). Peak-to-peak intensity is indicated. Receiver gain for BCC and SK was 630 and 500, respectively. Other EPR conditions were the same. EPR spectra were obtained using a single scan. The samples are described in the main text.

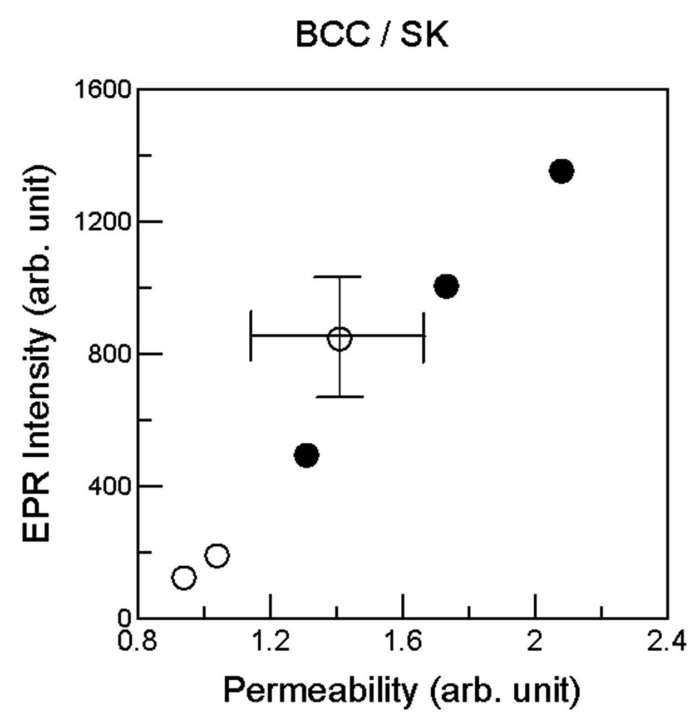

Fig. 3 Plots of electron paramagnetic resonance intensities versus permeability for seborrheic keratosis $(\mathrm{SK}, \boldsymbol{\bullet})$ and basal cell carcinoma $(\mathrm{BCC}, \mathrm{O})$ samples. The extended error bars were used due to the cutting out of a portion of the paraffin-embedded specimens.

lower magnetic field of the peak. The dotted line (the shoulder) corresponds to the shoulder signal overlap. On the basis of the previously reported spectra, it was concluded that the signal indicates the presence of pheomelanin radical in the MM sample. In the case of NP, we previously reported that the NP sample mostly contained eumelanin-related radicals, according to histology, EPR, and EPRI studies. ${ }^{5,8}$ Thus, if (C) is subtracted from (A), the lineshape and linewidth of the subtracted spectrum are similar to the previously reported values. ${ }^{4}$ Notably, the similar spectral intensity of the subtraction yields good results. Thus, the subtracted spectrum mostly shows a pheomelanin radical component (Fig. 4(D)). The pheomelanin-containing compounds also revealed wider EPR signals (approximately

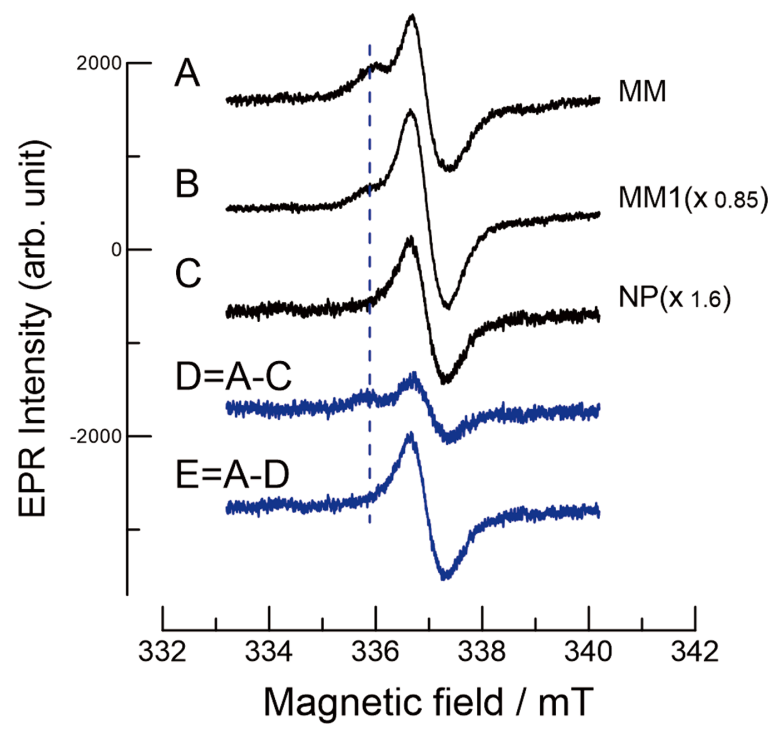

Fig. 4 Electron paramagnetic resonance spectra of (A) malignant melanoma (MM), (B) MM-1 (another melanoma sample), (C) nevus pigmentosus (NP), (D) subtracted spectrum of (C) from (A), and (E) subtracted spectrum of (D) from (A).

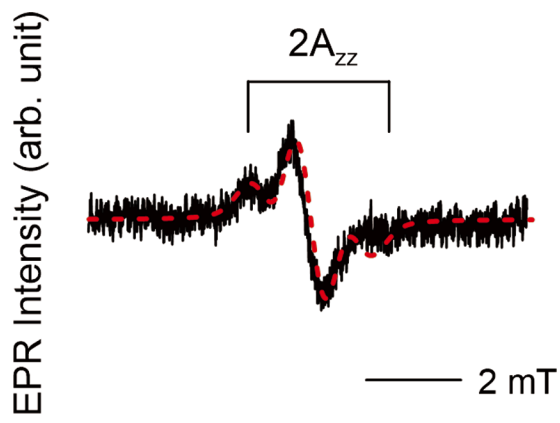

Fig. 5 An isotropic EPR simulation (red-color dotted line) of the subtracted spectrum of Fig. 4(D). We used the following parameters: linewith, $0.6 \mathrm{mT}$; nitrogen (14) hyperfine value, $1.51 \mathrm{mT}$. We added two spectra: one is single line and the other is nitrogen three lines with one-to-one ratio to reproduce the subtracted spectrum. The hyperfine coupling $\left(2 \mathrm{~A}_{\mathrm{zz}}\right)$ component is also indicated.

$0.6-0.7 \mathrm{mTs}$ ), compared with the singlet of synthetic dopa melanin. ${ }^{4}$

The hyperfine coupling $\left(2 \mathrm{~A}_{\mathrm{zz}}\right)$ component in Fig. 5 is approximately $3.1 \mathrm{mT}$, which is very similar to the reported value. $^{4}$ If (D) is subtracted from (A) in Fig. 4, the main component is the eumelanin radical (Fig. 4(E)). The perfect pheomelanin component can be difficult to obtain using the spectral subtraction method due to the signal-to-noise ratio. Moreover, the subtracted spectrum is similar to synthetic cysteinyldopa melanin. The fur of C57BL/6J $\left(A^{\mathrm{Y}} / a\right)$ mice, and fur of yellow gerbils exhibited EPR signals with clear hyperfine structure $\left(2 \mathrm{~A}_{\mathrm{zz}}\right.$, approximately $\left.3.1 \mathrm{mT}\right)$ connected to the location of unpaired electrons near the nucleus of ${ }^{14} \mathrm{~N} .{ }^{4}$

Figure 5 shows an EPR simulation of the subtracted spectrum of Fig. 4(D). In this simulation, we used a JEOL isotropic simulation program. We used two spectra: one was single line and the other was nitrogen three lines. We used the following parameters: linewith, $0.6 \mathrm{mT} ;{ }^{14} \mathrm{~N}$ hyperfine value, $1.51 \mathrm{mT}$. We added them in one-to-one ratio to reproduce the subtracted 


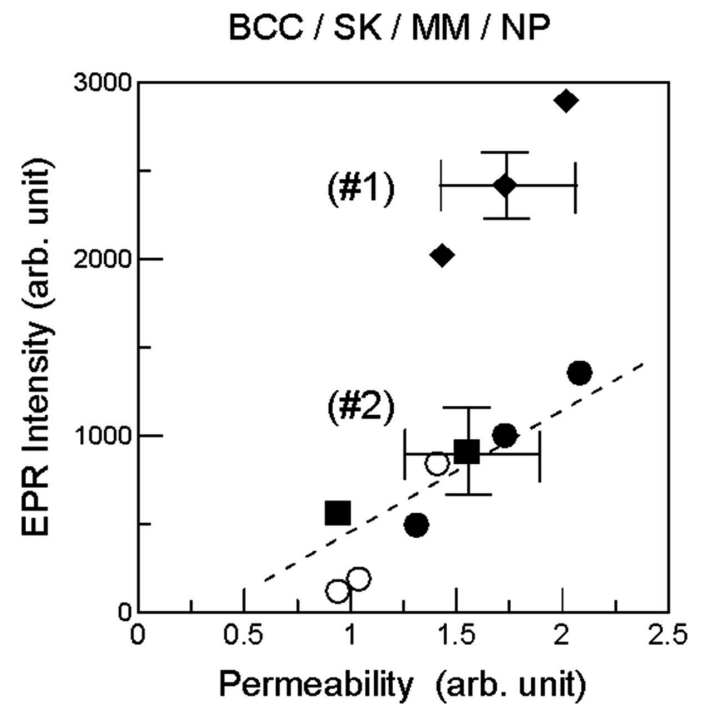

Fig. 6 Plot of electron paramagnetic resonance (EPR) intensities versus permeability for seborrheic keratosis (SK, O), basal cell carcinoma (BCC, $\bigcirc)$, malignant melanoma (MM, $\diamond)$, and nevus pigmentosus $(\mathrm{NP}, \mathbf{\square})$ samples. The MM points are above the dotted line. (\#1) and (\#2) correspond to the spectrum of Fig. 4(A) and 4(C), respectively. Extended error bars were used because of the cutting out of a portion of paraffin-embedded specimens.

spectrum. This simulation suggests that the unpaired electron interacts with nitrogen (14) and may belong to the pheomelanin molecule (Fig. 1). It is important to point out that the nitrogen hyperfine coupling $\left(2 \mathrm{~A}_{\mathrm{zz}}\right)$ is a little smaller than the subtracted one. The discrepancy of the simulated spectrum at a higher magnetic field may be due to the immobilized molecular motion of the radical. In order to simulate the immobilized molecule, it requires having six principal values $\left(\mathrm{A}_{\mathrm{XX}}, \mathrm{A}_{\mathrm{YY}}, \mathrm{A}_{\mathrm{ZZ}}, \mathrm{g}_{\mathrm{XX}}, \mathrm{g}_{\mathrm{YY}}\right.$, and $\mathrm{g}_{\mathrm{zz}}$ ). The principal values can be obtained by the knowledge of the exact molecular structure of pheomelanin.

Figure 6 shows the plot of EPR intensities versus permeability for SK $(\bullet), \mathrm{BCC}(\bigcirc), \mathrm{MM}(\bullet)$, and NP $(\boldsymbol{\square})$ samples. However, the MM points are above the dotted line. Most of the points are along the fitted line, which can be related to the eumelanin (dark color) radicals in the samples. If we subtract the eumelanin radicals, the EPR spectrum will mostly indicate pheomelanin radicals. In other words, if we subtract pheomelanin radicals from the MM spectrum, the EPR intensity of MM will decrease.

It is a useful calculation of the degree of linear relation between EPR and permeability. We tried to rule out the relation for SK, BCC, NP, and MM. The correlation coefficient of approximately 0.8 in the $n=8$ set of data can be considered to indicate the high degree of relationship in the range. However, the MM data are off the fitted line, mainly because of an additional overlap, such as pheomelanin radical. If the signal of $\mathrm{MM}$ is subtracted from NP, an additional pheomelanin radical appears.

Moreover, pheomelanin has been reported to be present in human red hair ${ }^{6}$ and animal hair. ${ }^{4}$ In humans, pheomelanin is involved in the development of skin cancer and/or increased risk for skin cancer. ${ }^{6}$ In addition, it was reported that cysteinyldopa was found in large amounts in the plasma and urine of patients with malignant melanoma. ${ }^{10}$ Cysteinyldopa is believed to be formed by the rapid enzymatic hydrolysis of 5-S-glutathionedopa found in melanin-producing cells. For these the reasons, it suggested as a marker in the diagnosis and the detection of the
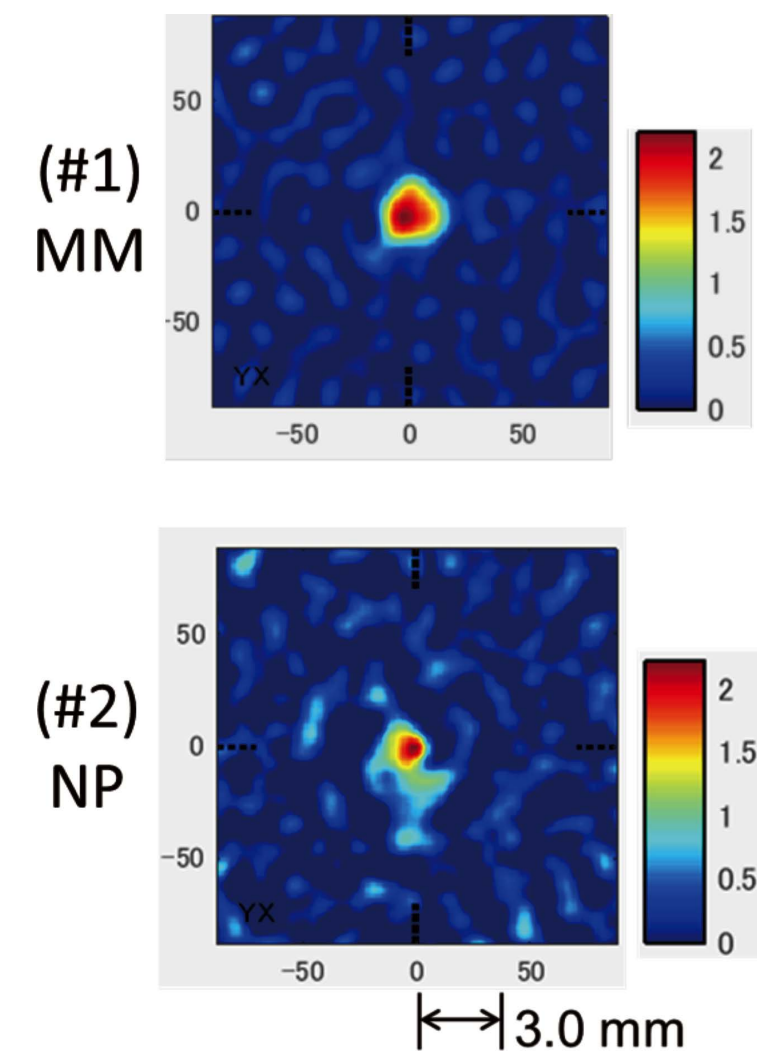

Fig. 7 Electron paramagnetic resonance images of paraffinembedded malignant melanoma (MM) and nevus pigmentosus (NP) specimens. (\#1) The red colored section of an MM case is recognized. (\#2) The red colored section of an NP case is recognized. The samples are described in the main text. (\#1) and (\#2) correspond to the spectrum of Fig. 4(A) and 4(C), respectively.

melanoma. ${ }^{10}$ Pheomelanin radicals were also reported in paraffinembedded MM samples. 5,7 Thus, the present analyses suggest that pheomelanin can be related to the malignancy of melanoma.

Figure 7 depicts the EPR images showing paraffin-embedded MM and NP specimens. The most intensely colored section of an MM case (\#1) as well as the least intensely colored section of an NP case (\#2) is recognized. The EPR images show that the paraffin-embedded $\mathrm{MM}$ is above the eumelanin radical line. Other intensely colored sections of MM are also recognized. The large red color is related to the strong radical signal in the sample. The distribution corresponds to the pigmented region. However, current EPRI is not able to differentiate two species in the MM cases at this time.

The strong signal of EPR and the red region of EPRI are caused by the high radical concentration (pheomelanin) in the MM samples. On the other hand, the results for NP show values that are approximately two times smaller. The background of EPRI for NP has background noise due to weak EPR signal. It is noteworthy that the EPRI data process is a linear process so that any superposition will come up with the same ratio considering modulation based acquisition and the integral of the signal. The EPRI results are consistent with the EPR results.

\section{Conclusions}

We obtained the linear correlation between permeability value and EPR intensity for pigmented specimens in the study region, 
except for MM. In addition, we performed the isotropic simulation in order to obtain more detailed information about the melanin-related radical. The 2D EPRI supports the pigment results. Further analyses showed that pheomelanin radical involvement was proposed for MM. The present spectroscopic analyses suggest that the pheomelanin radical may exist in malignant melanoma

\section{Acknowledgements}

The authors thank Dr. Toru Osanai (Graduate School of Health Sciences at Hirosaki University) for his help with permeability measurements. Part of this research was supported by KAKENHI Grant number 18K19890 from the Japan Society for the Promotion of Science (JSPS) (K. N.).

\section{References}

1. M. Cichorek, M. Wachulska, A. Stasiewicz, and A. Tymińska, Postep. Derm. Alergol., 2013, 1, 30.

2. E. J. Land and P. A. Riley, Pigment. Cell Res., 2000, 13, 273.

3. A. Hennessy, C. Oh, and B. Diffey, Pigment. Cell Res., $\mathbf{2 0 0 5}, 18,220$.

4. A. Slominski, P. M. Plonka, A. Pisarchik, J. L. Smart, V. Tolle, J. Wortsman, and M. J. Low, Endocrinology, 2005, 146, 1245.

5. K. Nakagawa, S. Minakawa, D. Sawamura D, and H. Hara, Anal. Sci., 2017, 33, 1357.

6. E. Chikvaidze and I. Khachatryan, Int. J. Cosme. Sci., 2011, 33, 322

7. K. Nakagawa, S. Minakawa, and D. Sawamura, Anal. Sci., 2018, 34, 837.

8. K. Nakagawa, S. Minakawa, C. Itabashi, and D. Sawamura, Anal. Sci., 2019, 35, 265.

9. M. R. Chedekel, S. K. Smith, P. W. Post, A. Pokora, and D. L. Vessell, Proc. Natl. Acad. Sci. U. S. A., 1978, 75, 5395.

10. H. Goto, M. Usui, K. Wakamatsu, and S. Ito, Jpn. J. Ophthalmol., 2001, 45, 538.

11. K. Nakagawa, S. Minakawa, and D. Sawamura, Hirosaki University Internal Review Board approval, 2014, 390.

12. K. Nakagawa, Lipids, 2010, 45, 91.

13. K. Nakagawa, S. Minakawa, and D. Sawamura, J. Dermatol. Sci., 2012, 65, 222.

14. http://epri.uchicago.edu or http://epr-it.specman4epr.com/ The web describes EPR-IT software.

15. L. Chauffe, J. J. Windle, and M. Friedman, Biophys. J., 1975, 15, 565. 